\title{
Segmented YSO scintillation detectors as a new $\beta$-implant detection tool for decay spectroscopy in fragmentation facilities
}

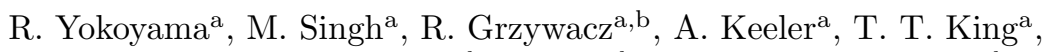 \\ J. Agramunt ${ }^{\mathrm{c}}$, N. T. Brewer ${ }^{\mathrm{b}, \mathrm{a}}$, S. Go ${ }^{\mathrm{d}}$, J. Heideman ${ }^{\mathrm{a}}$, J. Liu ${ }^{\mathrm{e}, \mathrm{d}}$, \\ S. Nishimura ${ }^{\mathrm{d}}$, P. Parkhurst ${ }^{\mathrm{f}}$, V. H. Phongg,d, M. M. Rajabalih ${ }^{\mathrm{h}}$, \\ B. C. Rasco ${ }^{\mathrm{b}, \mathrm{a}}$, K. P. Rykaczewski ${ }^{\mathrm{b}}$, D. W. Stracener ${ }^{\mathrm{b}}$, J. L. Tain ${ }^{\mathrm{c}}$, \\ A. Tolosa-Delgado ${ }^{c}$, K. Vaigneuri ${ }^{i}$, M. Wolińska-Cichocka ${ }^{j}$ \\ ${ }^{a}$ Department of Physics and Astronomy, University of Tennessee, Knoxville, TN 37996, \\ $U S A$ \\ ${ }^{b}$ Physics Division, Oak Ridge National Laboratory, Oak Ridge, TN 37830, USA \\ ${ }^{c}$ Instituto de Fisica Corpuscular (CSIC-Universitat de Valencia), E-46071 Valencia, Spain \\ ${ }^{d}$ RIKEN, Nishina Center, 2-1 Hirosawa, Wako, Saitama 351-0198, Japan \\ ${ }^{e}$ Department of Physics, the University of Hong Kong, Pokfulam Road, Hong Kong \\ ${ }^{f}$ Proteus, Inc., Chagrin Falls, OH 44022, USA \\ ${ }^{g}$ Faculty of Physics, VNU University of Science, 334 Nguyen Trai, Thanh Xuan, Hanoi, \\ Vietnam \\ ${ }^{h}$ Department of Physics, Tennessee Technological University, Cookeville, TN 38505, USA \\ ${ }^{i}$ Agile Technologies, Knoxville, TN 37932 \\ ${ }^{j}$ Heavy Ion Laboratory, University of Warsaw, Warsaw PL-02-093, Poland
}

\begin{abstract}
A newly developed segmented YSO scintillator detector was implemented for the first time at the RI-beam Factory at RIKEN Nishina Center as an implantationdecay counter. The results from the experiment demonstrate that the detector is a viable alternative to conventional silicon-strip detectors with its good timing resolution and high detection efficiency for $\beta$ particles. A Position-Sensitive Photo-Multiplier Tube (PSPMT) is coupled with a $48 \times 48$ segmented YSO crystal. To demonstrate its capabilities, a known short-lived isomer in ${ }^{76} \mathrm{Ni}$ and the $\beta$ decay of ${ }^{74}$ Co were measured by implanting those ions into the YSO detector. The half-lives and $\gamma$-rays observed in this work are consistent with the known values. The $\beta$-ray detection efficiency is more than $80 \%$ for the decay of ${ }^{74} \mathrm{Co}$.
\end{abstract}

The study of $\beta$-decays far from stability is essential to understand the evolu- 
tion of nuclear structure and nucleosynthesis processes. It has been shown that, $\beta$-decay properties of neutron-rich nuclei heavier than iron impact $r$-process calculations that determine the solar abundance of elements [1, 2, Typically, $\beta$-decay experiments with such exotic nuclei involve intense cocktail beam from fragmentation facilities. The role of an implantation detector in these experiments is to measure the energy and the positions of both heavy ion implantation and $\beta$-ray emission in order to correlate the identified ion with $\beta$-decay events. Conventional implantation detectors developed for the beams at fragmentation facilities use double-sided silicon-strip detectors (DSSDs) such as AIDA [3] or WAS3ABi [4] which were used recently with the BRIKEN [5, 6, 7] neutron counter array at the Radioactive Ion Beam Factory (RIBF).

The idea of a new implantation detector using segmented scintillator crystals was proposed previously [8, aiming to use it as a fast $\beta$ trigger for the neutron time-of-flight (ToF) array, VANDLE [9]. Energy information of $\beta$-delayed neutrons can be deduced from neutron ToF measurements [10]. The DSSDs cannot be used for this application because of their slow response. The new detector constructed using scintillator crystal is expected to enable neutron ToF measurements at avanced fragmentation facilities that can provide high-intensity exotic beams. A prototype plastic scintillator based detector described in Ref. [8] was used as the fast trigger for timing investigations at the NSCL [11]. Plastic detectors suffer from low stopping power for $\beta$ particles which adversely impacts the ability to make the ion-decay correlations.

In this paper, development of a new implantation detector using segmented YSO (Yttrium Orthosilicate, $\mathrm{Y}_{2} \mathrm{SiO}_{5}$ ) crystals is presented. There are several advantages of using YSO. It offers better timing response than DSSDs and better energy resolution compared to plastic scintillators. The effective atomic number $(Z=35)$ and the density $\left(4.4 \mathrm{~g} / \mathrm{cm}^{3}\right)$ of the YSO crystal are higher than silicon, resulting in higher stopping power, and therefore leading to shorter range of $\beta$ particles emitted by the ions implanted into the detector. Due to these properties, YSO detectors are expected to have higher detection efficiency and better position resolution for high-energy $\beta$ rays than silicon-strip detectors. 
Another advantage of using YSO scintillators is that they can be used with higher implantation rates since they are radiation hard and the fast scintillation (42 ns decay time [12]) enables rapid re-triggering. The YSO is non-hygroscopic, relatively cheap and easy to make into pixelated arrays. The design presented here is very easy to implement in an experiment. The use of resistive readout requires only five detector signals for processing. The smaller light yield for heavy ions is typical for inorganic scintillators, as measured for example in Ref. [13, which reduces the required dynamic range of the light sensor needed to record signals induced both by $\beta$ particles and high-Z implants.

The segmented YSO detector was implemented for the first time at the RIBF to measure $\beta$ decays of nuclei around and beyond ${ }^{78} \mathrm{Ni}$. Analysis and results on the isomer of ${ }^{76} \mathrm{Ni}$ and the $\beta$ decay of ${ }^{74} \mathrm{Co}$ nuclei are presented as a demonstration of the capability of this new implantation detector. The $\beta$ efficiency for the ${ }^{74} \mathrm{Co}$ decay and $\gamma$-ray absorption for the ${ }^{76} \mathrm{Ni}$ isomer are also shown.

(a)

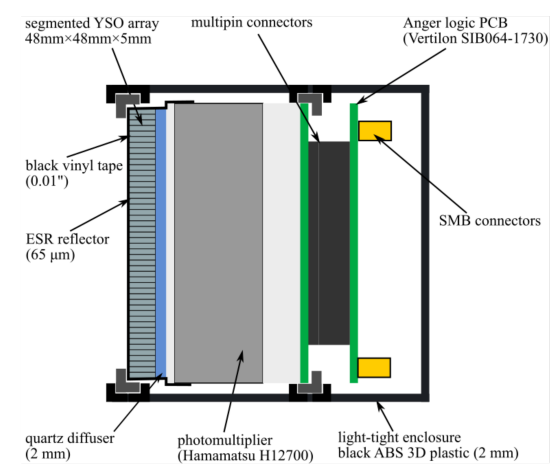

(b)

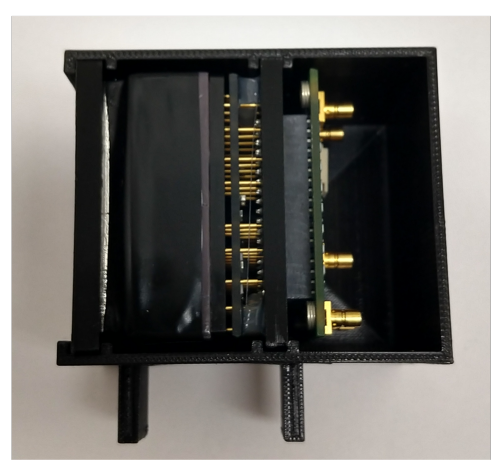

Figure 1: Schematic drawing (a) and a picture (b) of the YSO detector device. Detailed dimensions of the H12700 photomultiplier can be found on its data sheet [14. Schematic of the Anger-logic can be found in 15.

The detector unit consists of a segmented YSO crystal coupled to a flat panel multi-anode photomultiplier by Hamamatsu, H12700. The YSO scintillator is a 
high-granularity matrix of $1 \times 1 \mathrm{~mm}$ segments arranged into a $48 \times 48$ array in the $x-y$ plane. The isolation of the pixel segments of the array using $65 \mu \mathrm{m}$ ESR (3M) reflector material enhances the position resolution, the sides, as well as the face of the crystal, also have the ESR covering to prevent scintillation light loss. The thickness of the crystal is $5 \mathrm{~mm}$ which is designed to stop all the ions of interest in the ${ }^{78} \mathrm{Ni}$ region produced by in-flight fission of ${ }^{238} \mathrm{U}$ at the RIBF. A $2 \mathrm{~mm}$ thick quartz light diffuser between the YSO crystal and the PSPMT was required in order to achieve sub-anode position resolution by spreading the light from one pixel onto several anodes of the PSPMT. The detector unit was enclosed in a 3D-printed light-tight box with a thin light shielding black vinyl tape covering the face of the detector. The 64 anode channels of the photomultiplier are read out by a resistive-network, also known as Anger-logic [16]. In this implementation, Vertilon SIB064-1730 (a custom version of the SIB064B-1018 [15]) was used as an Anger-logic read-out board. The board is connecting the 64 anode outputs with a resistor network and is read out from its four corners, which enables $x$ - and $y$-position reconstruction from ratios of the signal amplitudes. The dynode signal is used to determine the time and the total energy. Each of the four anode signals and the dynode are split into channels with two different gain settings. One set of signals is amplified 20 times by a high bandwidth amplifier for $\beta$-ray counting and the other is for heavy-ion implantations.

The YSO implantation detector was implemented together with the BRIKEN neutron counter system [5, 6] at RIKEN, RIBF in order to study $\beta$ decays of the nuclei around and beyond ${ }^{78} \mathrm{Ni}$. The neutron-rich nuclei are produced by in-flight fission of a primary ${ }^{238} \mathrm{U}^{86+}$ beam with an energy of $345 \mathrm{MeV} /$ nucleon, induced at a $4 \mathrm{~mm}$ thick ${ }^{9}$ Be production target. The typical intensity of the primary beam was $\sim 60 \mathrm{pnA}$ during the run. Fission fragments are separated and identified in the BigRIPS in-flight separator [17] on an event-by-event basis by their proton numbers $(Z)$ and the mass-to-charge ratio $(A / Q)$. These quantities are obtained by the measurement of the $B \rho$, time of flight (TOF), and energy loss $(\Delta E)$ in BigRIPS. A detailed explanation of the particle identification at 
the BigRIPS is found in Ref. [18, 19]. There were $2 \times 10^{5}{ }^{76} \mathrm{Ni}$ and $1.5 \times 10^{4}$ ${ }^{74}$ Co ions implanted into the YSO detector in $\sim 140$ hours of run.

The secondary beam was transported to the final focal plane and implanted into the YSO detector. Four layers of the double-sided silicon-strip detector (DSSD) WAS3ABi 4 were installed upstream of the YSO detector. The DSSD consists of sixteen $3 \mathrm{~mm}$ wide strips in both the $x$ and $y$ directions. The typical rate of the ion implantation in YSO during the run was $\sim 60 \mathrm{cps}$.

The YSO and WAS3ABi detectors are placed at the center of the BRIKEN high-density polyethylene moderator [5]. The BRIKEN detector was operated in hybrid mode, which is composed of 140 proportional counters filled with ${ }^{3} \mathrm{He}$ gas for neutron detection [5] and two ORNL clover-type HPGe detectors [20] for high-resolution $\gamma$-ray detection.

As a result of the implementation of the YSO detector at the RIBF, we obtained position images for $\beta$ and implantation events in the detector and successfully achieved the correlation between recoil and decay events. The $x-y$ image for $\beta$ events in the YSO detector is shown in Fig. 2(a). Each dot in the plot corresponds to a $1 \times 1 \mathrm{~mm}$ segment of the YSO crystal. The horizontal dip along $y \sim 0.5$ is caused by the structure of the YSO segmentation. The array is made of two parts of $24 \times 48$ segmented crystals and the ESR between those two in the middle distorts the image due to modification of light sharing between the two pieces. The image has the resolution sufficient to distinguish segments for most of the active region. Figure 2 (b) shows the image of the implantation events occurring in the $1 \mathrm{~s}$ before $\beta$ events observed in one of the segments marked with a red circle in Fig. 2 (a). There is a clear peak on top of randomly correlated ion implantation events which are spread over the entire detector surface. The full-width half-maximum of the peak corresponding to the correlated implants shown in Fig. 2 (d) is $1.3 \mathrm{~mm}$.

In ${ }^{76} \mathrm{Ni}$, an isomeric state is reported by Mazzocchi et al. produced by fragmentation of a ${ }^{86} \mathrm{Kr}$ beam in a ${ }^{9} \mathrm{Be}$ target at NSCL 21. Figure 3 shows the delayed $\gamma$-ray spectrum after ${ }^{76} \mathrm{Ni}$ implantation in the YSO detector. The energy of the four $\gamma$-ray peaks agrees with the literature [21]. The half-life of 

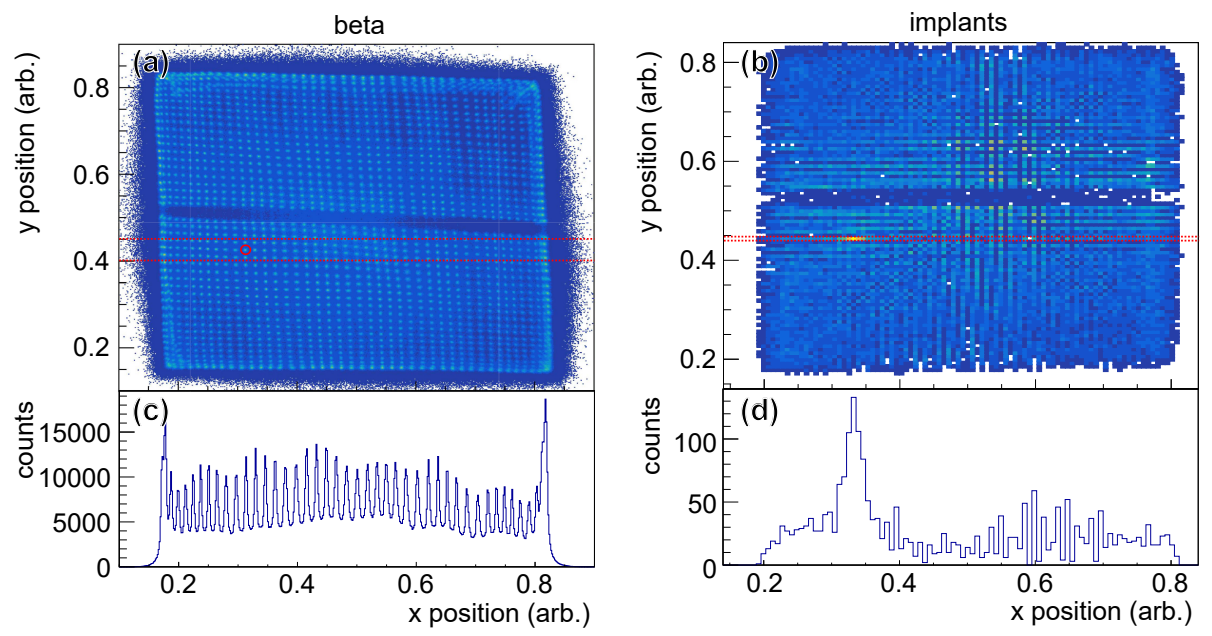

Figure 2: YSO $x$ - $y$ images of (a) $\beta$ events and (b) implantation events correlated to the $\beta$ events in a segment shown in the red circle in panel (a). (c) and (d) are the projection of (a) and (b), respectively, on to the $x$ axis in the cut shown by the red dashed lines.

the isomer is obtained as 520(15) ns by fitting the decay curve of all the four $\gamma$-ray peaks, which is also consistent with the literature value, $590_{-110}^{+180} \mathrm{~ns}[21$.

Isomeric $\gamma$-ray spectra of ${ }^{76} \mathrm{Ni}$ implanted into YSO and WAS3ABi detectors are compared in order to verify the effects of $\gamma$-ray absorption in each detector material. YSO is expected to attenuate $\gamma$-rays more than silicon due to its higher effective atomic number and density. A simple simulation using Geant4 was performed to assess the severity of the absorption in YSO. The results for the point source placed $6 \mathrm{~cm}$ apart from the surface of the clover detector showed that the detection efficiency of $140 \mathrm{keV} \gamma$-rays dropped by a factor of four when the source is exactly placed at the center of the YSO crystal and when YSO is placed perpendicular to the clover-detector surface. However, in our experiment, we expected less absorption since the ion implantation is spread over the crystal and there is effectively less material thickness than in the simulation. For the case of the measured decay of ${ }^{76} \mathrm{Ni}$ isomer, the ratio between the two peak areas, that of $143 \mathrm{keV}$ and $930 \mathrm{keV}$ is (49(8)) / (12(3)) $=4.3(15)$ for WAS3ABi, whereas the ratio for YSO is $(701(30)) /(191(14)))=$ 
3.7(3). This shows that absorption in YSO detector of the $143 \mathrm{keV} \gamma$-ray with respect to the $930 \mathrm{keV}$ line is only $1.2(4)$ times larger than that in WAS3ABi.

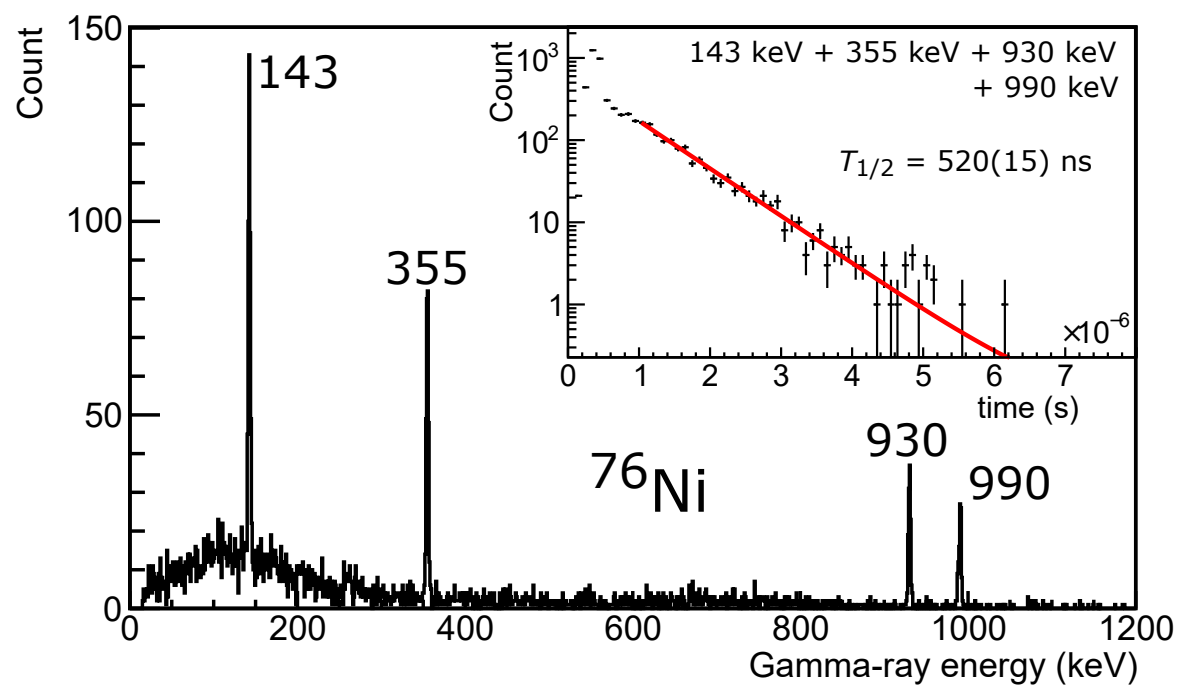

Figure 3: Delayed $\gamma$-ray spectrum of ${ }^{76} \mathrm{Ni}$ gated from $1.2 \mu$ s to $5 \mu$ s after the implantation. The time spectrum gated on the energy of all the four $\gamma$-ray peaks is plotted at the right top corner of the figure. The red line shows the fitting function with an exponential decay and a constant background.

$\beta-\gamma$ spectrum of ${ }^{74} \mathrm{Co}$ was first reported by Mazzocchi et al. [21, then by Go et al. 22, and recently by Morales et al. [23] with improved statistics. Xu et al. 24] and Hosmer et al. 25] have also reported the half-life of ${ }^{74} \mathrm{Co}$ decay. Hosmer et al. also measured $P_{n}$ branching ratio with $\mathrm{BF}_{3}$ neutron counters [25].

Figure 4 shows the decay curve of ${ }^{74} \mathrm{Co} \beta$ events. The fit function includes the parent, daughter $\left({ }^{74} \mathrm{Ni}\right)$, grand-daughter $\left({ }^{74} \mathrm{Cu}\right)$, and neutron-daughter $\left({ }^{73} \mathrm{Ni}\right)$ decays along with a linear background. The half-lives of ${ }^{74} \mathrm{Ni},{ }^{74} \mathrm{Cu}$, and ${ }^{73} \mathrm{Ni}$ are fixed to the literature values, $0.5077 \mathrm{~s}$ [24], $1.59 \mathrm{~s}$ [26], and $0.84 \mathrm{~s}$ [27, respectively. The $P_{n}$ branching ratio of ${ }^{74} \mathrm{Co}$ is fixed to $18 \%$ [25]. The ${ }^{74} \mathrm{Co}$ halflife obtained in this work is $30.8(6) \mathrm{ms}$, which is consistent with the literature values, 30(11) $\mathrm{ms}$ [25] and 31.6(15) ms 24. A $\beta$-decay branch from isomeric state in ${ }^{74} \mathrm{Co}$ is reported by Morales et al. [23] with a $28(3) \mathrm{ms}$ half-life. 


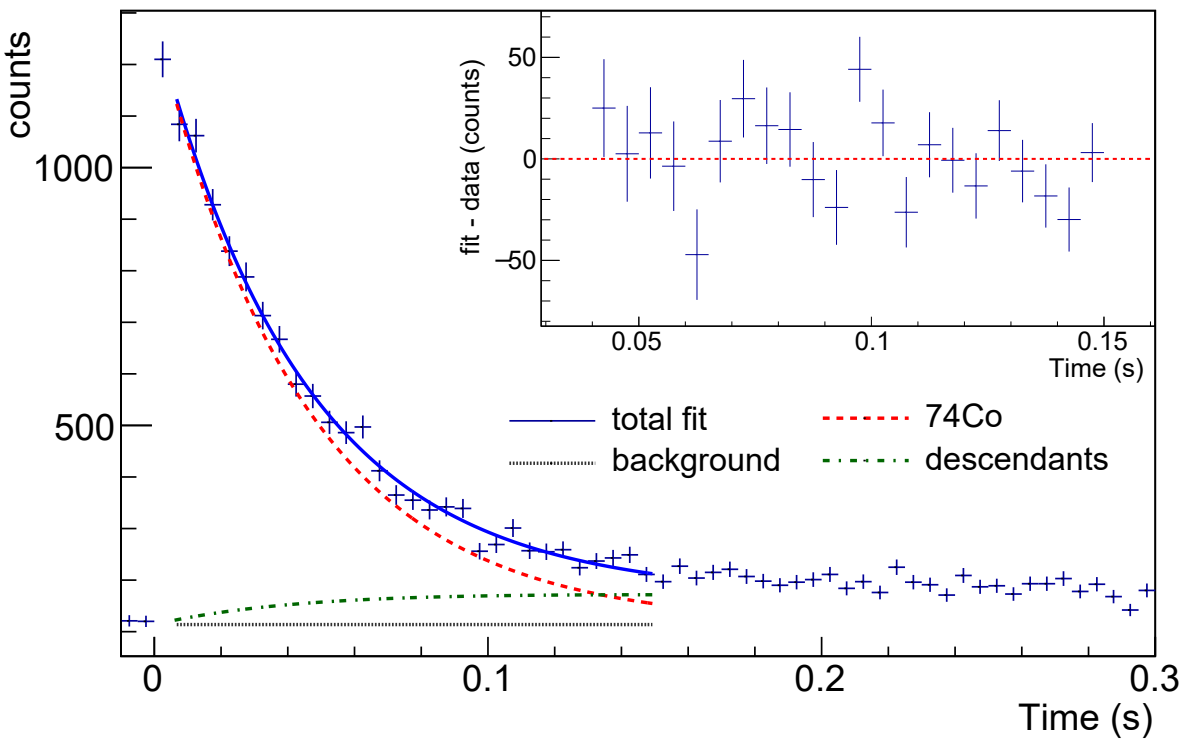

Figure 4: The decay curve of the $\beta$ rays from ${ }^{74}$ Co decays. The solid blue curve represents the fitting function. The dashed red curve shows the decay component of the parent nucleus, ${ }^{74} \mathrm{Co}$. The dashed-dotted green curve is the sum of the daughter and neutron-daughter branches. The dotted black line shows the linear background. The plot at the right top of the figure shows the difference between the data points and the fitting function.

Figure 5 shows the $\gamma$-ray spectrum of ${ }^{74}$ Co decay detected within $100 \mathrm{~ms}$ after implantation. All the $\gamma$-rays in our spectrum are consistent with the reported values in Ref. [22, 23]. We also observed $\gamma$-rays at 2588 and $3611 \mathrm{keV}$ which are reported as decays from the isomeric state in ${ }^{74} \mathrm{Co}[22,23]$.

The main purpose of using the segmented YSO detector in this experiment was to demonstrate and exploit its high $\beta$-detection efficiency. Figure 6 shows the $\beta$-efficiency curve as a function of the maximum correlation distance between $\beta$ events and implant events. The correlation distance is calibrated to $\mathrm{mm}$ from the fact that the distance between two adjacent segments is $1 \mathrm{~mm}$. The $\beta$ efficiency is calculated by dividing the integral of the parent decay curve obtained from fitting by the total number of ${ }^{74} \mathrm{Co}$ implants. The main source of the dead time is due to blocking $\beta$ triggers for $200 \mu$ s after ion implantation in order to wait until the signal recovers from the large pulse from heavy ions. 

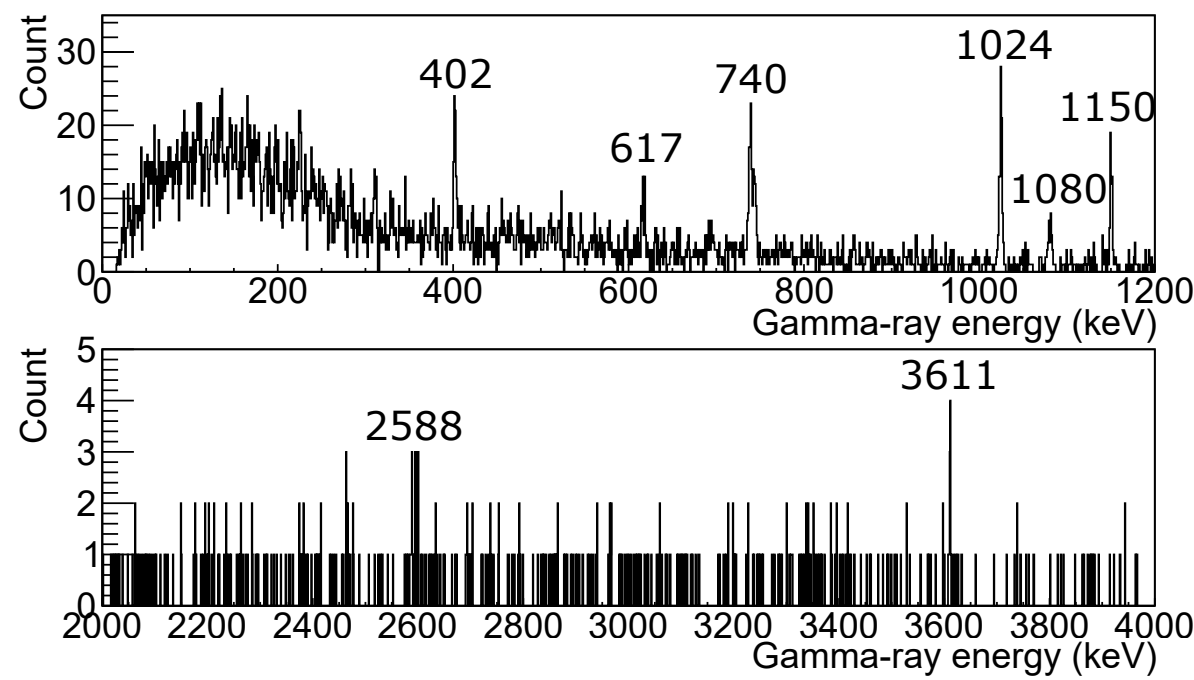

Figure 5: The $\gamma$-ray spectrum from the $\beta$ decays of ${ }^{74} \mathrm{Co}$. Energies of the peaks decaying by a similar half life to that of ${ }^{74} \mathrm{Co}(\sim 30 \mathrm{~ms})$ are labeled in $\mathrm{keV}$.

This corresponds to $1.2 \%$ dead time when the implantation rate is $60 \mathrm{cps}$. The dead time of the data acquisition system during the run is neglected since it was much smaller than $1 \%$. The $\beta$ efficiency of the YSO detector for ${ }^{74} \mathrm{Co}$ decay is more than $80 \%$ when with maximum correlation distance grater than $3 \mathrm{~mm}$, as shown in Fig. 6. This correlation range can be linked to the energy of the $\beta$ particles in YSO.

In summary, we implemented the new segmented YSO detector for the first time in a $\beta$-decay experiment at RIBF, RIKEN. As a demonstration, data on the isomer in ${ }^{76} \mathrm{Ni}$ and the $\beta$ decay of ${ }^{74} \mathrm{Co}$ are shown. The implant- $\beta$ correlation by the YSO detector was successful and obtained 30.8(6) ms half-life for ${ }^{74} \mathrm{Co}$, which is consistent with previous reports [24, 25]. We also confirmed the $\gamma$ rays of the ${ }^{74} \mathrm{Co}$ decay reported in Ref. 22, 23. The $\gamma$-ray absorption in YSO is affecting the $\gamma$-ray detection efficiency, but not more than by a factor of $1.2(4)$ at $143 \mathrm{keV}$, compared to the silicon-strip detector, WAS3ABi. The $\beta$-ray detection efficiency for ${ }^{74} \mathrm{Co}$ is $\sim 80 \%$ with a $3 \mathrm{~mm}$ correlation radius. We have demonstrated that a compact and simple-to-operate segmented YSO detector 


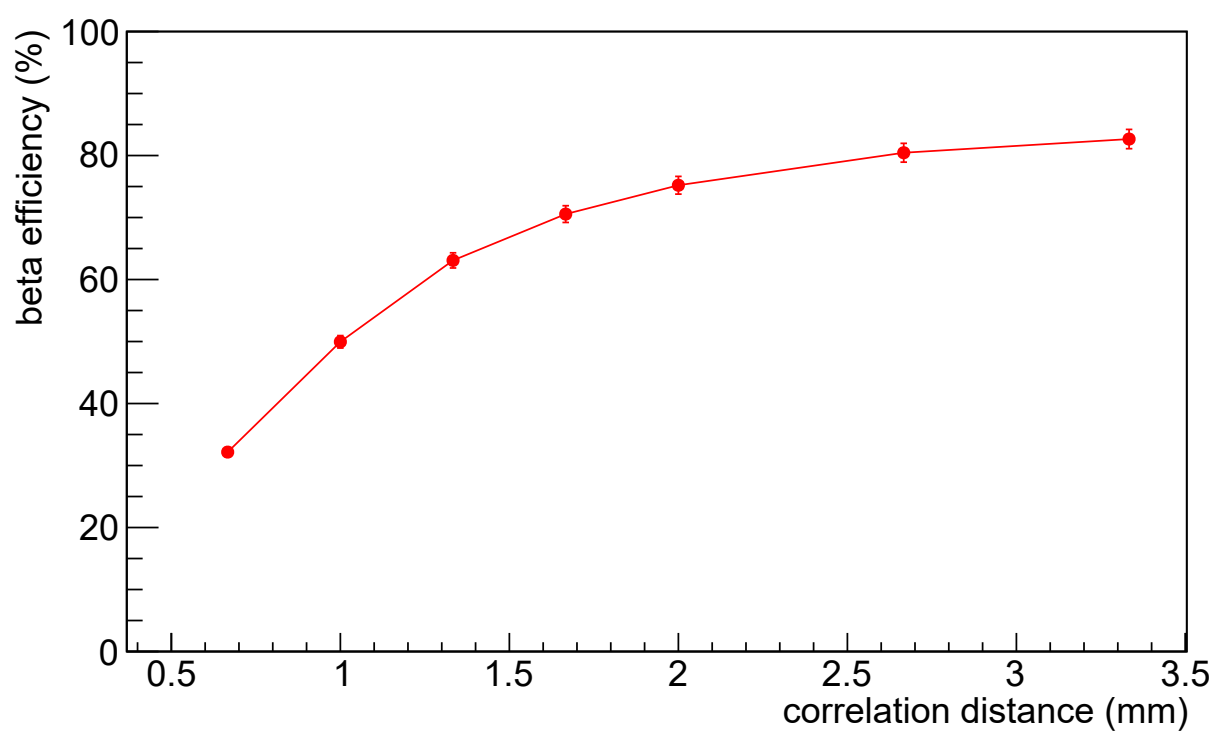

Figure 6: The efficiency curve of the $\beta$ rays from ${ }^{74}$ Co decays as a function of correlation distances between $\beta$ events and implant events.

can be used as a very high-efficiency $\beta$-implant and ion- $\gamma$ detection tool. Also, a high stopping power equivalent to eight layers of 1-mm DSSDs makes it a much cheaper option to use in ion-implantation experiments particularly when the range straggling of implanted ions requires the use of a lot of active stopping material. The segmented YSO crystal is in the same price range of a single DSSD detector. The limited resolution of the scintillator does not impede $\beta$ decay experiments. In the situation when ions are fully stripped, the limited energy resolution for ion energy measurement is also not a limitation. YSO and other similar inorganic scintillators are known to be extremely radiation hard and can be reused in many experiments. The high stopping power for $\beta$ particles resulted in very high detection efficiency. Together with its excellent sub-nanosecond timing resolution, the YSO detector can be, in many cases, a better alternative to DSSD detectors for $\beta$-decay experiments at fragmentation facilities. 


\section{acknowledgments}

The present experiment was carried out at the RI Beam Factory operated by RIKEN Nishina Center, RIKEN and CNS, University of Tokyo. This research was supported in part by the Office of Nuclear Physics, U.S. Department of Energy under Award No. DE-FG02-96ER40983 (UTK).

\section{References}

\section{References}

[1] M. R. Mumpower, G. C. McLaughlin, R. Surman, Formation of the rareearth peak: Gaining insight into late-time r-process dynamics, Physical Review C 85 (4) (2012) 045801. arXiv:1109.3613, doi:10.1103/PhysRevC. 85.045801 .

[2] R. Surman, M. Mumpower, A. Aprahamian, The Sensitivity of r -Process Nucleosynthesis to Individual $\beta$-Delayed Neutron Emission Probabilities, in: JPS Conference Proceedings, Vol. 6, Tokyo, Japan, 2015, p. 010010. doi:10.7566/JPSCP.6.010010.

[3] C. J. Griffin, T. Davinson, A. Estrade, D. Braga, I. Burrows, P. ColemanSmith, T. Grahn, A. Grant, L. J. Harkness-Brennan, M. Kogimtzis, I. Lazarus, S. Letts, Z. Liu, G. Lorusso, K. Matsui, S. Nishimura, R. D. Page, M. Prydderch, V. Pucknell, S. Rinta-Antila, O. Roberts, D. A. Seddon, J. Simpson, J. Strachan, S. L. Thomas, P. J. Woods, ??-decay studies of r-process nuclei using the Advanced Implantation Detector Array (AIDA), Proceedings of Science NIC XIII (2014) 097.

[4] S. Nishimura, G. Lorusso, Z. Xu, J. Wu, R. Gernh, H. S. Jung, Y. K. Kwon, Z. Li, K. Steiger, H. Sakurai, WAS3ABi : The beta-counting system for the EURICA project, RIKEN Accelerator Progress Report 46 (2013) 182. 
[5] A. Tarifeño-Saldivia, J. L. Tain, C. Domingo-Pardo, F. Calviño, G. Cortés, V. H. Phong, A. Riego, J. Agramunt, A. Algora, N. Brewer, R. CaballeroFolch, P. J. Coleman-Smith, T. Davinson, I. Dillmann, A. Estradé, C. J. Griffin, R. Grzywacz, L. J. Harkness-Brennan, G. G. Kiss, M. Kogimtzis, M. Labiche, I. H. Lazarus, G. Lorusso, K. Matsui, K. Miernik, F. Montes, A. I. Morales, S. Nishimura, R. D. Page, Z. S. Podolyák, V. F. Pucknell, B. C. Rasco, P. Regan, B. Rubio, K. P. Rykaczewski, Y. Saito, H. Sakurai, J. Simpson, E. Sokol, R. Surman, A. Svirkhin, S. L. Thomas, A. Tolosa, P. Woods, Conceptual design of a hybrid neutron-gamma detector for study of $\beta$-delayed neutrons at the RIB facility of RIKEN, Journal of Instrumentation 12 (2017) P04006. doi:10.1088/1748-0221/12/04/P04006.

[6] A. Tolosa-Delgado, J. Agramunt, J. Tain, A. Algora, C. Domingo-Pardo, A. Morales, B. Rubio, A. Tarifeo-Saldivia, F. Calvio, G. Cortes, N. Brewer, B. Rasco, K. Rykaczewski, D. Stracener, J. Allmond, R. Grzywacz, R. Yokoyama, M. Singh, T. King, M. Madurga, S. Nishimura, V. Phong, S. Go, J. Liu, K. Matsui, H. Sakurai, G. Kiss, T. Isobe, H. Baba, S. Kubono, N. Fukuda, D. Ahn, Y. Shimizu, T. Sumikama, H. Suzuki, H. Takeda, P. Sderstrm, M. Takechi, C. Bruno, T. Davinson, C. Griffin, O. Hall, D. Kahl, P. Woods, P. Coleman-Smith, M. Labiche, I. Lazarus, P. Morrall, V. E. Pucknell, J. Simpson, S. Thomas, M. Prydderch, L. Harkness-Brennan, R. Page, I. Dillmann, R. Caballero-Folch, Y. Saito, A. Estrade, N. Nepal, F. Montes, G. Lorusso, J. Liang, S. Bae, J. Ha, B. Moon, Commissioning of the briken detector for the measurement of very exotic $\beta$-delayed neutron emitters, Nuclear Instruments and Methods in Physics Research Section A: Accelerators, Spectrometers, Detectors and Associated Equipment 925 (2019) 133 - 147. doi:https://doi.org/10.1016/j.nima.2019.02.004.

URL http://ww.sciencedirect.com/science/article/pii/ S0168900219301743

[7] R. Yokoyama, et al., submitted to Phys. Rev. Lett. 
[8] M. Alshudifat, R. Grzywacz, S. V. Paulauskas, Development of a Segmented Scintillator for Decay Studies, Physics Procedia 66 (2015) 445-450. doi: $10.1016 / j \cdot$ phpro.2015.05.056.

URL http://dx.doi.org/10.1016/j.phpro.2015.05.056

[9] W. A. Peters, S. Ilyushkin, M. Madurga, C. Matei, S. V. Paulauskas, R. K. Grzywacz, D. W. Bardayan, C. R. Brune, J. Allen, J. M. Allen, Z. Bergstrom, J. Blackmon, N. T. Brewer, J. A. Cizewski, P. Copp, M. E. Howard, R. Ikeyama, R. L. Kozub, B. Manning, T. N. Massey, M. Matos, E. Merino, P. D. O'Malley, F. Raiola, C. S. Reingold, F. Sarazin, I. Spassova, S. Taylor, D. Walter, Performance of the Versatile Array of Neutron Detectors at Low Energy (VANDLE), Nuclear Instruments and Methods in Physics Research, Section A: Accelerators, Spectrometers, Detectors and Associated Equipment 836 (2016) 122-133. doi: 10.1016/j.nima.2016.08.054.

URL http://dx.doi.org/10.1016/j.nima.2016.08.054

[10] M. Madurga, S. V. Paulauskas, R. Grzywacz, D. Miller, D. W. Bardayan, J. C. Batchelder, N. T. Brewer, J. A. Cizewski, A. Fijałkowska, C. J. Gross, M. E. Howard, S. V. Ilyushkin, B. Manning, M. Matoš, A. J. Mendez, K. Miernik, S. W. Padgett, W. A. Peters, B. C. Rasco, A. Ratkiewicz, K. P. Rykaczewski, D. W. Stracener, E. H. Wang, M. Wolińska-Cichocka, E. F. Zganjar, Evidence for Gamow-Teller Decay of Ni 78 Core from BetaDelayed Neutron Emission Studies, Physical Review Letters 117 (9) (2016) 092502. doi:10.1103/PhysRevLett.117.092502.

[11] B. Crider, C. Prokop, S. Liddick, M. Al-Shudifat, A. Ayangeakaa, M. Carpenter, J. Carroll, J. Chen, C. Chiara, H. David, A. Dombos, S. Go, R. Grzywacz, J. Harker, R. Janssens, N. Larson, T. Lauritsen, R. Lewis, S. Quinn, F. Recchia, A. Spyrou, S. Suchyta, W. Walters, S. Zhu, Shape coexistence from lifetime and branching-ratio measurements in 68,70ni, Physics Letters B 763 (2016) 108 - 113. doi:https://doi.org/10.1016/j.physletb.2016.10.020 
URL http://www.sciencedirect.com/science/article/pii/ S0370269316305913

[12] C. L. Melcher, J. S. Schweitzer, C. A. Peterson, R. A. Manente, H. Suzuki, Inorganic Scintillators and Their Applications, Delft University Press, 1996.

[13] Y. Koba, H. Iwamoto, K. Kiyohara, T. Nagasaki, G. Wakabayashi, Y. Uozumi, N. Matsufuji, Scintillation Efficiency of Inorganic Scintillators for Intermediate-Energy Charged Particles, Progress in Nuclear Science and Technology 1 (2015) 218-221. doi:10.15669/pnst.1.218.

[14] Hamamatsu Photonics K.K., (accessed on May 6, 2019).

URL https://www.hamamatsu.com/resources/pdf/etd/ H12700\{_\}TPMH1348E.pdf

[15] Vertilon Corporation, (accessed on May 6, 2019).

URL http://vertilon.com/pdf/PS2738.pdf

[16] H. O. Anger, Scintillation camera, Review of Scientific Instruments 29 (1) (1958) 27-33. doi:10.1063/1.1715998.

[17] T. Kubo, In-flight RI beam separator BigRIPS at RIKEN and elsewhere in Japan, Nuclear Instruments and Methods in Physics Research Section B: Beam Interactions with Materials and Atoms 204 (2003) 97-113. doi : 10.1016/S0168-583X (02)01896-7.

URL http://linkinghub.elsevier.com/retrieve/pii/ S0168583X02018967

[18] T. Ohnishi, T. Kubo, K. Kusaka, A. Yoshida, K. Yoshida, M. Ohtake, N. Fukuda, H. Takeda, D. Kameda, K. Tanaka, N. Inabe, Y. Yanagisawa, Y. Gono, H. Watanabe, H. Otsu, H. Baba, T. Ichihara, Y. Yamaguchi, M. Takechi, S. Nishimura, H. Ueno, A. Yoshimi, H. Sakurai, T. Motobayashi, T. Nakao, Y. Mizoi, M. Matsushita, K. Ieki, N. Kobayashi, K. Tanaka, Y. Kawada, N. Tanaka, S. Deguchi, Y. Satou, Y. Kondo, 
T. Nakamura, K. Yoshinaga, C. Ishii, H. Yoshii, Y. Miyashita, N. Uematsu, Y. Shiraki, T. Sumikama, J. Chiba, E. Ideguchi, A. Saito, T. Yamaguchi, I. Hachiuma, T. Suzuki, T. Moriguchi, A. Ozawa, T. Ohtsubo, M. a. Famiano, H. Geissel, A. S. Nettleton, O. B. Tarasov, D. P. Bazin, B. M. Sherrill, S. L. Manikonda, J. a. Nolen, Identification of $45 \mathrm{New}$ Neutron-Rich Isotopes Produced by In-Flight Fission of a $238 \mathrm{U}$ Beam at $345 \mathrm{MeV} /$ nucleon, Journal of the Physical Society of Japan 79 (7) (2010) 073201. doi:10.1143/JPSJ.79.073201.

URL http://jpsj .ipap.jp/link?JPSJ/79/073201/

[19] N. Fukuda, T. Kubo, T. Ohnishi, N. Inabe, H. Takeda, D. Kameda, H. Suzuki, Identification and separation of radioactive isotope beams by the BigRIPS separator at the RIKEN RI Beam Factory, Nuclear Instruments and Methods in Physics Research Section B: Beam Interactions with Materials and Atoms 317 (2013) 323-332. doi:10.1016/j.nimb.2013.08.048. URL http://linkinghub.elsevier.com/retrieve/pii/ S0168583X13009890

[20] C. J. Gross, T. N. Ginter, D. Shapira, W. T. Milner, J. W. McConnell, A. N. James, J. W. Johnson, J. Mas, P. F. Mantica, R. L. Auble, J. J. Das, J. L. Blankenship, J. H. Hamilton, R. L. Robinson, Y. A. Akovali, C. Baktash, J. C. Batchelder, C. R. Bingham, M. J. Brinkman, H. K. Carter, R. A. Cunningham, T. Davinson, J. D. Fox, A. Galindo-Uribarri, R. Grzywacz, J. F. Liang, B. D. MacDonald, J. MacKenzie, S. D. Paul, A. Piechaczek, D. C. Radford, A. V. Ramayya, W. Reviol, D. Rudolph, K. Rykaczewski, K. S. Toth, W. Weintraub, C. Williams, P. J. Woods, C. H. Yu, E. F. Zganjar, Performance of the Recoil Mass Spectrometer and its detector systems at the Holifield Radioactive Ion Beam Facility, Nuclear Instruments and Methods in Physics Research, Section A: Accelerators, Spectrometers, Detectors and Associated Equipment 450 (1) (2000) 12-29. doi:10.1016/S0168-9002(00)00159-5.

[21] C. Mazzocchi, R. Grzywacz, J. C. Batchelder, C. R. Bingham, D. Fong, 
J. H. Hamilton, J. K. Hwang, M. Karny, W. Krolas, S. N. Liddick, A. F. Lisetskiy, A. C. Morton, P. F. Mantica, W. F. Mueller, K. P. Rykaczewski, M. Steiner, A. Stolz, J. A. Winger, Low energy structure of even-even $\mathrm{Ni}$ isotopes close to78Ni, Physics Letters, Section B: Nuclear, Elementary Particle and High-Energy Physics 622 (1-2) (2005) 45-54. doi:10.1016/ j.physletb.2005.07.006.

[22] S. Go, et al., Gamow-Teller transformation of ${ }^{74}$ Co and decay properties of ${ }^{78} \mathrm{Co} \rightarrow{ }^{78} \mathrm{Ni}$, submitted to Phys. Rev. C.

[23] A. Morales, G. Benzoni, H. Watanabe, G. de Angelis, S. Nishimura, L. Coraggio, A. Gargano, N. Itaco, T. Otsuka, Y. Tsunoda, P. V. Isacker, F. Browne, R. Daido, P. Doornenbal, Y. Fang, G. Lorusso, Z. Patel, S. Rice, L. Sinclair, P.-A. Söderström, T. Sumikama, J. Valiente-Dobón, J. Wu, Z. Xu, A. Yagi, R. Yokoyama, H. Baba, R. Avigo, F. B. Garrote, N. Blasi, A. Bracco, A. Bruce, F. Camera, S. Ceruti, F. Crespi, M.-C. Delattre, Z. Dombradi, A. Gottardo, T. Isobe, I. Kojouharov, N. Kurz, I. Kuti, S. Lalkovski, K. Matsui, B. Melon, D. Mengoni, T. Miyazaki, V. ModamioHoybjor, S. Momiyama, D. Napoli, M. Niikura, R. Orlandi, Z. Podolyák, P. Regan, H. Sakurai, E. Sahin, D. Sohler, H. Schaffner, R. Taniuchi, J. Taprogge, Z. Vajta, O. Wieland, M. Yalcinkaya, Is seniority a partial dynamic symmetry in the first vg9/2 shell?, Physics Letters B 781 (2018) 706 - 712. doi:https://doi.org/10.1016/j.physletb.2018.04.049.

URL http://www.sciencedirect.com/science/article/pii/ S0370269318303435

[24] Z. Y. Xu, S. Nishimura, G. Lorusso, F. Browne, P. Doornenbal, G. Gey, H. S. Jung, Z. Li, M. Niikura, P. A. Söderström, T. Sumikama, J. Taprogge, Z. Vajta, H. Watanabe, J. Wu, A. Yagi, K. Yoshinaga, H. Baba, S. Franchoo, T. Isobe, P. R. John, I. Kojouharov, S. Kubono, N. Kurz, I. Matea, K. Matsui, D. Mengoni, P. Morfouace, D. R. Napoli, F. Naqvi, H. Nishibata, A. Odahara, E. Sahin, H. Sakurai, H. Schaffner, I. G. Stefan, D. Suzuki, R. Taniuchi, V. Werner, Decay half-lives of Co 76,77, Ni 79,80, 
and $\mathrm{Cu}$ 81: Experimental indication of a doubly magic Ni 78, Physical Review Letters 113 (3) (2014) 032505. doi:10.1103/PhysRevLett.113. 032505

[25] P. Hosmer, H. Schatz, A. Aprahamian, O. Arndt, R. R. C. Clement, A. Estrade, K. Farouqi, K. L. Kratz, S. N. Liddick, A. F. Lisetskiy, P. F. Mantica, P. Möller, W. F. Mueller, F. Montes, A. C. Morton, M. Ouellette, E. Pellegrini, J. Pereira, B. Pfeiffer, P. Reeder, P. Santi, M. Steiner, A. Stolz, B. E. Tomlin, W. B. Walters, A. Wöhr, Half-lives and branchings for $\beta$-delayed neutron emission for neutron-rich $\mathrm{Co}-\mathrm{Cu}$ isotopes in the r-process, Physical Review C 82 (2) (2010) 025806. arXiv:1011.5255, doi:10.1103/PhysRevC.82.025806

[26] J. A. Winger, J. C. Hill, F. K. Wohn, E. K. Warburton, R. L. Gill, A. Piotrowski, D. S. Brenner, Structure of neutron-rich Zn74 from Cu74 decay and shell-model calculations for even-A Zn nuclei, Physical Review C 39 (5) (1989) 1976-1984. doi:10.1103/PhysRevC.39.1976.

[27] S. Franchoo, M. Huyse, K. Kruglov, Y. Kudryavtsev, W. F. Mueller, R. Raabe, I. Reusen, P. Van Duppen, J. Van Roosbroeck, L. Vermeeren, A. Wöhr, H. Grawe, K. L. Kratz, B. Pfeiffer, W. B. Walters, Monopole migration in $69,71,73 \mathrm{Cu}$ observed from $\beta$ decay of laser-ionized $68-74 \mathrm{Ni}$, Physical Review C 64 (5) (2001) 054308. doi:10.1103/PhysRevC.64.054308. 